\title{
First Order Smooth Composite Chebyshev Finite Difference Method for Solving Coupled Lane- Emden Problem in Catalytic Diffusion Reactions
}

\author{
Soner Aydinlik ${ }^{\mathrm{a}, *}$, Ahmet Kiris ${ }^{\mathrm{b}}$ \\ ${ }^{a}$ Department of Software Engineering, Faculty of Engineering, Dogus University, 34469, \\ Istanbul, Turkey \\ saydinlikedogus.edu.tr \\ ${ }^{b}$ Department of Mathematics, Faculty of Science and Letters, Istanbul Technical University, \\ 34469, Istanbul, Turkey
}

(Received January 26, 2021)

\begin{abstract}
A new effective technique based on Chebyshev Finite Difference Method is introduced. First order smoothness of the approximation polynomial at the end points of each sub-interval is imposed in addition to the continuity condition. Both round-off and truncation error analyses are given besides the convergence analysis. Coupled Lane Emden boundary value problem in Catalytic Diffusion Reactions is investigated by using presented method. The obtained results are compared with the existing methods in the literature and it is observed that the proposed method gives more reliable results than the others.
\end{abstract}

\section{Introduction}

Lane-Emden equations [1,2] illustrate many physical phenomena such as astrophysics problems, temperature variation, reaction diffusion process and heat conduction in human head [3-5]. The general system form of nonlinear Lane-Emden equations with the mixed type of boundary conditions are given as

\footnotetext{
${ }^{*}$ Corresponding author.
} 


$$
\left\{\begin{array}{l}
u^{\prime \prime}(x)+\frac{r}{x} u^{\prime}(x)=f_{1}(u(x), v(x)) \\
v^{\prime \prime}(x)+\frac{r}{x} v^{\prime}(x)=f_{2}(u(x), v(x)), \\
u^{\prime}(0)=0, \quad u(1)=\beta_{1}, \quad v^{\prime}(0)=0, \quad v(1)=\beta_{2},
\end{array}\right.
$$

where $r, \beta_{1}, \beta_{2}$ are the real constants and $f_{1}(u(x), v(x))$ and $f_{2}(u(x), v(x))$ are the real valued functions. Equation (1.1) models the catalytic diffusion reactions [6]. There are several numerical methods as well as analytical methods to deal with these types of equations in recent years [6-16].

As a non-uniform scheme, Chebyshev Finite Difference Method (CFDM) has been widely used for the solution of initial or boundary value problems for the last two decades especially [8, 1720]. Due to the known advantages of Chebyshev polynomials such as orthogonality and economization, both recursive relations between itself and its derivatives can be obtained easily and they have the lowest maximum error in the given range compared to the other polynomial approximations of the same order. The approximation polynomial is expected to satisfy the problem at the collocation points (Chebyshev Gauss-Lobatto nodes) in CFDM and thus, the nonlinear differential equations with conditions reduce to the nonlinear system of the equations. In this study, we proposed a new technique, Smooth Composite CFDM based on splitting the overall interval into subinterval in CFDM. The main difference and the advantage of the presented method from both CFDM and Composite CFDM is $\mathrm{C}^{1}$-smoothness at the end points of the subintervals. We also performed both convergence and error analyses of the present method. We applied the presented method for the coupled Lane-Emden equations in Catalytic Diffusion Reactions and it is observed that the proposed method gives more reliable results than the existing methods in the literature.

\section{Smooth composite Chebyshev finite difference method}

An approximation polynomial to the solution of an initial or boundary value problem is given as,

$$
y(x)=\sum_{n=0}^{N} " a_{n} T_{n}(x)
$$


where, the superscript (") in the sum symbol means that half of the first and the last terms have to be taken and $N$ shows the order of the approximation polynomial. Here, Chebyshev polynomials are defined as

$$
T_{n}(x)=\cos (n \arccos (x)), \quad \forall n \geq 0, \quad x \in[-1,1] .
$$

By using the orthogonality, the unknown $a_{n}$ coefficients can be found as

$$
a_{n}=\frac{2}{N} \sum_{j=1}^{N} y\left(x_{j}\right) T_{n}\left(x_{j}\right)
$$

The Composite CFDM is an extension of the CFDM and based on dividing the given interval into the subintervals [21]. In Composite CFDM, the given interval $[a, b]$ is divided into the $M$-subinterval with the step size $h=\frac{(b-a)}{M}$. Then, the Gauss-Lobatto points $x_{k}=\cos \left(\frac{k \pi}{N}\right), \quad k=0,1, \ldots, N$ are transformed to the subintervals $\left[\frac{i(b-a)}{M}, \frac{(i+1)(b-a)}{M}\right]$ by

$$
t_{k i}=\frac{(b-a)}{2 M}\left(x_{k}+2 i+1\right)
$$

for each $i$. The approximation polynomial for the $i^{\text {th }}$ interval is given as

$$
y_{i}(x)=\sum_{n=0}^{N} " a_{i n} T_{n}(x),
$$

where

$$
a_{i n}=\frac{2}{N} \sum_{j=1}^{N} " y_{i}\left(x_{j}\right) T_{n}\left(x_{j}\right)
$$

The value of the $m^{\text {th }}$ order derivative of $y_{i}(x)$ in (2.5) at the points $x_{k}$ is given by

$$
y_{i}^{(m)}\left(x_{k}\right)=\sum_{j=0}^{N} d_{k, j}^{(m)} y_{i}\left(x_{j}\right)
$$

where,

$$
d_{k, j}^{(m)}=\frac{2}{N} \sum_{n=0}^{N} " T_{n}\left(x_{j}\right) T_{n}^{(m)}\left(x_{k}\right) .
$$

The first two of these coefficients are given as [22] 


$$
\begin{gathered}
d_{k, j}^{(1)}=\frac{4 \theta_{j}}{N} \sum_{n=0}^{N} \sum_{\substack{l=0 \\
(n+l) \text { odd }}}^{n-1} \frac{n \theta_{n}}{c_{l}} T_{n}\left(x_{j}\right) T_{l}\left(x_{k}\right), \quad k, j=0,1, \ldots, N, \\
d_{k, j}^{(2)}=\frac{2 \theta_{j}}{N} \sum_{n=0}^{N} \sum_{\substack{l=0 \\
(n+l) \text { even }}}^{n-2} \frac{n\left(n^{2}-l^{2}\right) \theta_{n}}{c_{l}} T_{n}\left(x_{j}\right) T_{l}\left(x_{k}\right), \quad k, j=0,1, \ldots, N
\end{gathered}
$$

where

$$
\left.\begin{array}{l}
\theta_{0}=\theta_{n}=1 / 2, \theta_{j}=1 \\
c_{0}=2, c_{i}=1
\end{array}\right\} \quad j=1,2, \ldots, N-1, \quad i \geq 1
$$

Here, we develop a new technique based on Composite CFDM method as follows:

In Composite CFDM method the interval $[a, b]$ is divided into the subintervals $\left[\frac{i(b-a)}{M}, \frac{(i+1)(b-a)}{M}\right]$. Then, the value of the approximation polynomial at the left end point of the interval must be equal to the value of the polynomial at the right end point of the preceding interval to satisfy continuity, i.e,

$$
y_{i+1}\left(x_{N}\right)=y_{i}\left(x_{0}\right)
$$

In Smooth Composite CFDM, the equality of the values of first order derivatives at the same points is added to (2.12) in order to satisfy first order smoothness, i.e,

$$
y_{i+1}^{\prime}\left(x_{N}\right)=y_{i}^{\prime}\left(x_{0}\right) \text {. }
$$

Writing the given initial or boundary value problem in terms of the approximation polynomial and its derivatives, the problem is transformed to the set of linear or nonlinear algebraic equations. Each subinterval contains a set of system of equations consisting of $(N+1)$ equations with $(N+1)$ unknowns of the approximation polynomial. Consequently, a system with $M \times(N+1)$ equations and $M \times(N+1)$ unknowns are obtained for the whole interval. After the solution of this system by using one of the root finding algorithms, the corresponding inverse transformation of (2.4) is applied to the obtained solutions for each subinterval. These results are used first in (2.6) and then in (2.5), thus the approximation polynomial, i.e, $P_{N}(x) \in C^{1}[a, b]$ is obtained by combining the approximation polynomials $P_{N}^{i}(x) \in C^{\infty}\left[\frac{i(b-a)}{M}, \frac{(i+1)(b-a)}{M}\right]$ for each interval, $i=0, \ldots, M-1$. 


\section{Convergence and error analyses of smooth composite CFDM}

The convergence of the Smooth Composite CFDM is proven and round off and truncation error analyses are given.

Theorem 3.1. (convergence)

Suppose $y_{i}(x) \in L_{w}^{2}(-1,1)$ with bounded by the second derivative, i.e, $\left|y_{i}^{\prime \prime}(x)\right| \leq C_{i}$, then its Chebyshev expansion converges to the $y_{i}(x)$ uniformly, that is the limiting case of (2.5), when $N$ goes to the infinity. Thus, $\bigcup_{i=1}^{M} y_{i}(x)$ also converges to the $y(x)$ on the whole interval.

Proof: Since set of Chebyshev polynomials are a complete orthogonal set in the Hilbert space $L_{w}^{2}[-1,1]$, multiplying both sides of (2.5) by $T_{m}(x)$ and the weight function and then integrating it over each subinterval $(\forall i=1,2,3, \ldots$ ) yields

$$
\int_{-1}^{1} w(x) T_{m}(x) y_{i}(x) d x=\int_{-1}^{1} \sum_{n=0}^{\infty} " w(x) T_{m}(x) a_{i n} T_{n}(x) d x .
$$

By the orthogonality property and the uniform convergence gives

$$
a_{i m}=\frac{2}{\pi c_{m}} \int_{-1}^{1} w(x) y_{i}(x) T_{m}(x) d x .
$$

Substituting $t=\cos \theta$ for $m>1$, we get

$$
a_{i m}=\frac{2}{\pi c_{m}} \int_{0}^{\pi} y_{i}(\cos \theta) \cos m \theta d \theta
$$

After applying integration by parts twice, we get

$$
\begin{aligned}
a_{i m} & =\left.\frac{2}{\pi c_{m}} y_{i}(\cos \theta) \frac{\sin m \theta}{m}\right|_{0} ^{\pi}+\frac{2}{m \pi c_{m}} \int_{0}^{\pi} y_{i}^{\prime}(\cos \theta) \sin m \theta \sin \theta d \theta \\
& =\left.\frac{2}{m \pi c_{m}} y_{i}^{\prime}(\cos \theta)\left(\frac{\sin (m-1) \theta}{m-1}-\frac{\sin (m+1) \theta}{m+1}\right)\right|_{0} ^{\pi} \\
+ & \frac{2}{m \pi c_{m}} \int_{0}^{\pi} y_{i}^{\prime \prime}(\cos \theta)\left(\frac{\sin (m-1) \theta}{m-1}-\frac{\sin (m+1) \theta}{m+1}\right) \sin \theta d \theta .
\end{aligned}
$$

The first part of (3.4) gives zero, and by using the triangle inequality for the last part, an upper bound for the coefficients is obtained as 


$$
\begin{aligned}
\left|a_{i m}\right| & =\left|\frac{2}{m \pi c_{m}} \int_{0}^{\pi} y_{i}^{\prime \prime}(\cos \theta)\left(\frac{\sin (m-1) \theta}{m-1}-\frac{\sin (m+1) \theta}{m+1}\right) \sin \theta d \theta\right| \\
& \leq \frac{2}{m \pi c_{m}} \int_{0}^{\pi}\left|y_{i}^{\prime \prime}(\cos \theta)\left(\frac{\sin (m-1) \theta}{m-1}-\frac{\sin (m+1) \theta}{m+1}\right) \sin \theta\right| d \theta \\
& \leq \frac{2 C_{i}}{m \pi c_{m}} \int_{0}^{\pi}\left|\left(\frac{\sin (m-1) \theta}{m-1}-\frac{\sin (m+1) \theta}{m+1}\right) \sin \theta\right| d \theta \\
& \leq \frac{2 C_{i}}{m \pi c_{m}} \int_{0}^{\pi}\left(\left|\frac{\sin (m-1) \theta}{m-1} \sin \theta\right|+\left|\frac{\sin (m+1) \theta}{m+1} \sin \theta\right|\right) d \theta \leq \frac{4 C_{i}}{\left(m^{2}-1\right) c_{m}} .
\end{aligned}
$$

for $m>1$. From (3.3), it is obvious that the coefficients $a_{i m}$ are also bounded for $m=0,1$. Considering $\left|T_{n}(x)\right| \leq 1$ and using the triangle inequality again, the approximation function can be written as,

$$
\left|y_{i}(x)\right|=\left|\sum_{m=0}^{\infty} " a_{i m} T_{m}(x)\right| \leq \sum_{m=0}^{\infty} "\left|a_{i m}\right| \leq\left|a_{i 0}\right|+\left|a_{i 1}\right|+\sum_{m=2}^{\infty} " \frac{4 C_{i}}{\left(m^{2}-1\right) c_{m}}<\infty .
$$

Equation (3.6) shows the absolute convergence of each approximation functions for each interval. Then, for the combined solution for whole interval

$$
|y(x)| \leq \max _{1 \leq i \leq M}\left|y_{i}(x)\right| \leq \max _{1 \leq i \leq M}\left(\left|a_{i 0}\right|+\left|a_{i 1}\right|+\sum_{m=2}^{\infty} "\left|\frac{4 C_{i}}{\left(m^{2}-1\right) c_{m}}\right|\right) \leq\left(\left|a_{i 0}\right|+\left|a_{i 1}\right|+\sum_{m=2}^{\infty} "\left|\frac{4 C}{\left(m^{2}-1\right) c_{m}}\right|\right)<\infty .
$$

where $C=\max _{1 \leq i \leq M}\left|C_{i}\right|$. This shows us, the approximation series is absolutely convergent, hence it converges to the $y(x)$ uniformly.

Elbarbary and El-Sayed give the following theorem for the round-off error [23].

Theorem 3.2. The effect of the round off error on the elements $d_{k, j}^{(1)}$ and $d_{k, j}^{(2)}$ are bounded as,

$$
\left\{\begin{array}{l}
d_{k, j}^{(1)^{*}}-d_{k, j}^{(1)} \leq 4 \theta_{j}\left(\delta-\mathrm{O}\left(\frac{1}{N^{2}} \delta\right)\right)\left(\frac{N^{2}}{3}+\frac{1}{6}\right) \\
d_{k, j}^{(2)^{*}}-d_{k, j}^{(2)} \leq \frac{4 \theta_{j}}{3}\left(\delta-\mathrm{O}\left(\frac{1}{N^{2}} \delta\right)\right)\left(\frac{N^{4}}{5}-\frac{1}{5}\right)
\end{array} .\right.
$$

Canuto gives the truncation error for Chebyshev polynomial approximation [24]. Here, we give the generalization of Canuto's theorem to the composite CFDM as follows: 
Theorem 3.3. Suppose, the approximation of the function $y_{i}(x)$ for each subinterval is given by $P_{N} y_{i}=\sum_{m=0}^{N} a_{i m} T_{m}$. For $\forall y_{i}(x) \in H_{w}^{m}(-1,1), m \geq 0$, the truncation error estimate,

$$
\left\|y_{i}-P_{N} y_{i}\right\|_{L_{w}^{2}(-1,1)} \leq C_{i} N^{-m}\left\|y_{i}\right\|_{H_{w}^{m, N}(-1,1)}
$$

is satisfied.

Proof: From the properties of Chebyshev polynomials given in Section 2, it is easily to see,

$$
\left\|y_{i}\right\|_{L_{w}^{2}(-1,1)}^{2}=\frac{1}{2}\left\|y_{i}^{*}\right\|_{L_{w}^{2}(0,2 \pi)}^{2}
$$

which defines an isomorphism between $L_{w}^{2}(-1,1)$ and $L_{w}^{2}(0,2 \pi)$. Moreover, it maps $H_{w}^{m}(-1,1)$ into the space of $H_{p}^{m}(0,2 \pi)$, where $p$ indicates periodic functions. Thus, since $y_{i} \in C^{m-1}[-1,1]$ , by periodicity, $y_{i}^{*} \in C^{m-1}(-\infty, \infty)$. The absolute values of derivatives of cosines smaller than unit, we have

$$
\left\|y_{i}^{*}\right\|_{H^{m}(0,2 \pi)} \leq C_{i}\left\|y_{i}\right\|_{H_{w}^{m}(-1,1)}^{2}
$$

for $m \geq 1$. By defining the symmetric truncation of Fourier series up to the same degree, i.e. $N$ , $P_{N}^{*}\left(\sum_{k=-\infty}^{\infty} v_{k} e^{i k \theta}\right)=\sum_{k=-N}^{N} v_{k} e^{i k \theta}$. Since $y_{i}(x)=\sum_{m=0}^{\infty} a_{i m} T_{m}(x)$ and $y_{i}^{*}(\theta)=\sum_{m=0}^{\infty} a_{i m} \cos (k \theta)$, then $y_{j}^{*}(\theta)=\sum_{m=0}^{\infty} a_{j m} \frac{e^{i k \theta}+e^{-i k \theta}}{2}$, thus $\left(P_{N} y_{i}\right)^{*}=P_{N}^{*} y_{i}^{*} \forall y_{i} \in L_{w}^{2}(-1,1)$. Consequently,

$$
\left\|y_{i}-P_{N} y_{i}\right\|_{L_{w}^{2}(-1,1)}=\frac{1}{\sqrt{2}}\left\|y_{i}^{*}-P_{N}^{*} y_{i}^{*}\right\|_{L_{w}^{2}(0,2 \pi)} \leq N^{-m}\left|C_{i}\right|\left\|y_{i}^{*(m)}\right\|_{L^{2}(0,2 \pi)}^{2}
$$

Lastly,

$$
\begin{aligned}
& \left\|y-P_{N} y\right\|_{L_{w}^{2}(-1,1)} \leq\left\|\bigcup_{i=1}^{M}\left(y_{i}-P_{N} y_{i}\right)\right\|\left\|_{L_{w}^{2}(-1,1)} \leq \max _{1 \leq i \leq M}\right\|\left(y_{i}-P_{N} y_{i}\right) \|_{L_{w}^{2}(-1,1)} \\
& \leq \max _{1 \leq i \leq M} N^{-m}\left|C_{i}\right|\left\|y_{i}^{*(m)}\right\|_{L^{2}(0,2 \pi)}^{2} \leq C N^{-m} \max _{1 \leq i \leq M}\left\|y_{i}^{*(m)}\right\|_{L^{2}(0,2 \pi)}^{2}
\end{aligned}
$$




\section{Numerical examples}

In this section, two nonlinear problems arising in chemical reactions are solved by Smooth Composite CFDM. These equations are coupled Lane Emden boundary value problems and do not have exact solutions. Instead, we investigate the maximum absolute residual errors of the equations,

$$
\begin{aligned}
& M R_{n}^{(1)}(x)=\max _{0 \leq x \leq 1}\left|u_{n}{ }^{\prime \prime}(x)+\frac{r}{x} u_{n}{ }^{\prime}(x)-f_{1}\left(u_{n}(x), v_{n}(x)\right)\right| \\
& M R_{n}^{(2)}(x)=\max _{0 \leq x \leq 1}\left|v_{n}{ }^{\prime \prime}(x)+\frac{r}{x} u_{n}{ }^{\prime}(x)-f_{2}\left(u_{n}(x), v_{n}(x)\right)\right|
\end{aligned} .
$$

Example 1: (Catalytic Diffusion Reaction Equation)

$$
\left\{\begin{array}{l}
u^{\prime \prime}(x)+\frac{2}{x} u^{\prime}(x)=a_{11} u^{2}(x)+a_{12} u(x) v(x), \\
v^{\prime \prime}(x)+\frac{2}{x} v^{\prime}(x)=a_{21} v^{2}(x)+a_{22} u(x) v(x), \\
u^{\prime}(0)=0, \quad u(1)=\beta_{1}, \quad v^{\prime}(0)=0, \quad v(1)=\beta_{2},
\end{array}\right.
$$

Here, $a_{11}=1, \quad a_{12}=\frac{2}{5}, \quad a_{21}=\frac{1}{2}, \quad \beta_{1}=1 \quad \beta_{2}=2$. In order to solve this problem by using Smooth Composite CFDM, the interval [0,1] is divided into the $M$ subintervals and each subinterval is transferred to $[-1,1]$. Then, applying Smooth Composite CFDM to the given differential equation and the boundary conditions, we have $M \times(2 N+2)$ nonlinear algebraic equations containing $M \times(2 N+2)$ unknown coefficients. Thus, the obtained system can be solved by any appropriate numerical method. Using the inverse transformations for each interval, the overall solution is obtained by combining the transformed solutions. The comparisons of the presented method with $[6,10,25]$ on the maximum residual errors are given in Table 1 and Table 2. 
Table 1. Comparisons on $M R_{n}^{(1)}(x)$ for example 1.

\begin{tabular}{|c|c|c|c|c|c|c|}
\hline$N$ & {$[25]$} & {$[6]$} & {$[9]$} & $M=1$ & $M=2$ & $M=4$ \\
\hline 2 & $7.55 E-02$ & $4.13 E-01$ & $3.61 E-01$ & $5.34 E-01$ & $2.88 E-01$ & $1.47 E-01$ \\
\hline 3 & $3.84 E-02$ & $2.36 E-01$ & $2.40 E-01$ & $1.94 E-01$ & $5.42 E-02$ & $1.45 E-02$ \\
\hline 4 & $1.74 E-02$ & $6.43 E-02$ & $4.21 E-02$ & $3.46 E-02$ & $6.00 E-03$ & $9.09 E-04$ \\
\hline 5 & $8.27 E-03$ & $4.79 E-02$ & $1.13 E-02$ & $7.25 E-03$ & $6.49 E-04$ & $5.17 E-05$ \\
\hline 6 & $3.78 E-03$ & $2.09 E-02$ & $1.73 E-03$ & $1.07 E-03$ & $5.77 E-05$ & $2.48 E-06$ \\
\hline 7 & $1.71 E-03$ & $1.09 E-02$ & $3.25 E-04$ & $1.75 E-04$ & $4.97 E-06$ & $1.12 E-07$ \\
\hline 8 & $7.19 E-04$ & $6.21 E-03$ & $4.58 E-05$ & $2.36 E-05$ & $3.89 E-07$ & $4.69 E-09$ \\
\hline 9 & $2.53 E-04$ & $3.35 E-03$ & $7.11 E-06$ & $3.36 E-06$ & $2.96 E-08$ & $1.89 E-10$ \\
\hline 10 & $6.27 E-05$ & $1.79 E-03$ & $9.47 E-07$ & $4.28 E-07$ & $2.14 E-09$ & $1.67 E-11$ \\
\hline 11 & $7.78 E-06$ & $9.61 E-03$ & $1.34 E-07$ & $5.56 E-08$ & $1.48 E-10$ & $1.52 E-11$ \\
\hline
\end{tabular}

Table 2. Comparisons on $M R_{n}^{(2)}(x)$ for example 1.

\begin{tabular}{|c|c|c|c|c|c|c|}
\hline$N$ & {$[25]$} & {$[6]$} & {$[9]$} & $M=1$ & $M=2$ & $M=4$ \\
\hline 2 & $1.77 E-01$ & $5.67 E-01$ & $8.36 E-01$ & $9.02 E-01$ & $6.71 E-01$ & $3.44 E-01$ \\
\hline 3 & $9.20 E-02$ & $3.09 E-01$ & $5.57 E-01$ & $4.56 E-01$ & $1.28 E-01$ & $3.47 E-02$ \\
\hline 4 & $4.27 E-02$ & $8.52 E-02$ & $1.10 E-01$ & $8.36 E-02$ & $1.46 E-02$ & $2.22 E-03$ \\
\hline 5 & $2.06 E-02$ & $6.09 E-02$ & $2.75 E-02$ & $1.77 E-02$ & $1.60 E-03$ & $2.15 E-04$ \\
\hline 6 & $9.54 E-03$ & $2.51 E-02$ & $4.31 E-03$ & $2.67 E-03$ & $1.45 E-04$ & $6.28 E-06$ \\
\hline 7 & $4.29 E-03$ & $1.36 E-02$ & $8.17 E-04$ & $4.42 E-04$ & $1.27 E-05$ & $2.88 E-07$ \\
\hline 8 & $1.73 E-03$ & $7.32 E-03$ & $1.17 E-04$ & $6.07 E-05$ & $1.00 E-06$ & $1.22 E-08$ \\
\hline 9 & $5.71 E-04$ & $3.28 E-03$ & $1.83 E-05$ & $8.71 E-06$ & $7.77 E-08$ & $4.64 E-10$ \\
\hline 10 & $1.28 E-04$ & $2.09 E-03$ & $2.47 E-06$ & $1.12 E-06$ & $5.68 E-09$ & $5.17 E-11$ \\
\hline 11 & $1.44 E-05$ & $1.11 E-03$ & $3.60 E-07$ & $1.47 E-07$ & $4.05 E-10$ & $7.32 E-12$ \\
\hline
\end{tabular}

Comparison of Smooth Composite CFDM with [6, 10, 25] we get better results. Moreover, the presented method, namely Smooth Composite CFDM gives more reliable results than CFDM as it can be easily seen from the Table 1 and Table 2. Absolute residual errors in logarithm of $u,\left(\log _{10}|e r r u|\right)$ and $v,\left(\log _{10} \mid\right.$ errv $\left.\mid\right)$ are given in Table 3 for the different values of $N$ and M . 
Table 3. The absolute residual errors of $u,\left(\log _{10}|e r r u|\right)$ and $v,\left(\log _{10}|\operatorname{err} v|\right)$ for the different values of $N$ and $M$.

4

\section{Example 2:}

This example describes the kinetics of the reaction between $\mathrm{CO}_{2}$ and phenyl glycidyl ether (PGE) in solution.

$$
\left\{\begin{array}{l}
u^{\prime \prime}(x)=\frac{\alpha_{1} u(x) v(x)}{1+\beta_{1} u(x)+\beta_{2} v(x)} \\
v^{\prime \prime}(x)=\frac{\alpha_{2} u(x) v(x)}{1+\beta_{1} u(x)+\beta_{2} v(x)}, \\
u(0)=1, \quad u(1)=k, \quad v^{\prime}(0)=0, \quad v(1)=1,
\end{array}\right.
$$

The functions $u(x)$ and $v(x)$ are the concentrations of $\mathrm{CO}_{2}$ and PGE. $x$ is the dimensionless distance as measured from the center and $k$ is the dimensionless concentration of $\mathrm{CO}_{2}$ at the surface of the catalyst. Here $\alpha_{1}=1, \alpha_{2}=2, \beta_{1}=1, \beta_{2}=3$ and $k=\frac{1}{2}$. 
Table 4. Comparisons on $M R_{n}^{(1)}(x)$ for example 2.

\begin{tabular}{|c|c|c|c|c|c|c|}
\hline$N$ & {$[25]$} & {$[6]$} & {$[9]$} & $M=1$ & $M=2$ & $M=4$ \\
\hline 1 & $4.15 E-02$ & 0.2 & 0.2 & 0.2 & 0.2 & 0.2 \\
\hline 2 & $5.28 E-03$ & $8.88 E-02$ & $3.86 E-02$ & $3.91 E-02$ & $2.00 E-02$ & $1.02 E-02$ \\
\hline 3 & $5.79 E-04$ & $8.88 E-03$ & $1.14 E-03$ & $1.63 E-03$ & $6.70 E-04$ & $2.04 E-04$ \\
\hline 4 & $2.09 E-04$ & $9.99 E-04$ & $4.87 E-04$ & $6.51 E-04$ & $9.70 E-05$ & $1.29 E-05$ \\
\hline 5 & $2.85 E-05$ & $8.88 E-05$ & $4.07 E-05$ & $5.51 E-05$ & $3.62 E-06$ & $2.34 E-07$ \\
\hline 6 & $5.45 E-06$ & $8.88 E-06$ & $1.01 E-06$ & $1.99 E-06$ & $1.40 E-07$ & $4.99 E-09$ \\
\hline 7 & $1.65 E-06$ & $8.88 E-07$ & $3.90 E-07$ & $6.21 E-07$ & $8.77 E-09$ & $1.58 E-10$ \\
\hline 8 & $2.81 E-06$ & $1.04 E-07$ & $4.88 E-09$ & $1.34 E-08$ & $3.85 E-10$ & $1.20 E-11$ \\
\hline 9 & $1.50 E-06$ & $2.76 E-08$ & $3.64 E-09$ & $6.61 E-09$ & $2.29 E-11$ & $9.65 E-12$ \\
\hline 10 & $4.95 E-07$ & $2.80 E-09$ & $1.22 E-10$ & $2.75 E-10$ & $2.67 E-12$ & $1.05 E-11$ \\
\hline
\end{tabular}

Table 5. Comparisons on $M R_{n}^{(2)}(x)$ for example 2.

\begin{tabular}{|l|c|c|c|c|c|c|}
\hline \multicolumn{1}{|c|}{$N$} & {$[25]$} & {$[6]$} & {$[9]$} & $M=1$ & $M=2$ & $M=4$ \\
\hline 1 & $8.31 E-02$ & 0.4 & 0.4 & 0.4 & 0.4 & 0.4 \\
\hline 2 & $1.05 E-02$ & $1.77 E-01$ & $7.73 E-02$ & $7.83 E-02$ & $4.00 E-02$ & $2.04 E-02$ \\
\hline 3 & $1.15 E-03$ & $1.77 E-02$ & $2.28 E-03$ & $3.26 E-03$ & $1.34 E-03$ & $4.09 E-04$ \\
\hline 4 & $4.18 E-04$ & $2.00 E-03$ & $9.75 E-04$ & $1.30 E-03$ & $1.94 E-04$ & $2.58 E-05$ \\
\hline 5 & $5.71 E-05$ & $1.77 E-04$ & $8.14 E-05$ & $1.10 E-04$ & $7.25 E-06$ & $4.69 E-07$ \\
\hline 6 & $1.08 E-05$ & $1.77 E-05$ & $2.03 E-06$ & $3.99 E-06$ & $2.81 E-07$ & $9.97 E-09$ \\
\hline 7 & $3.31 E-06$ & $1.77 E-06$ & $7.81 E-07$ & $1.24 E-06$ & $1.75 E-08$ & $3.13 E-10$ \\
\hline 8 & $5.69 E-06$ & $2.09 E-07$ & $9.76 E-09$ & $2.69 E-08$ & $7.69 E-10$ & $1.38 E-11$ \\
\hline 9 & $3.02 E-06$ & $5.52 E-08$ & $7.28 E-09$ & $1.32 E-08$ & $4.67 E-11$ & $8.58 E-12$ \\
\hline 10 & $9.91 E-07$ & $5.60 E-09$ & $2.43 E-10$ & $5.51 E-10$ & $1.16 E-12$ & $8.58 E-12$ \\
\hline
\end{tabular}

The comparisons on the maximum residual errors between $[6,10,25]$ and the present method are given in the Table 4 and Table 5. Also, the maximum residual errors obtained by the presented technique for the different number of subintervals $(M)$ and the different degrees of polynomials $(N)$ are given in the Table 6 and the absolute errors in logarithm (base 10) are given in the Table 6 for the different number of subintervals $(M)$ and the different degrees of polynomials ( $N$ ). 
Table 6. The absolute residual errors $u,\left(\log _{10}|e r r u|\right)$ and $v,\left(\log _{10}|e r r v|\right)$ for the different values of $M$ and $N$.

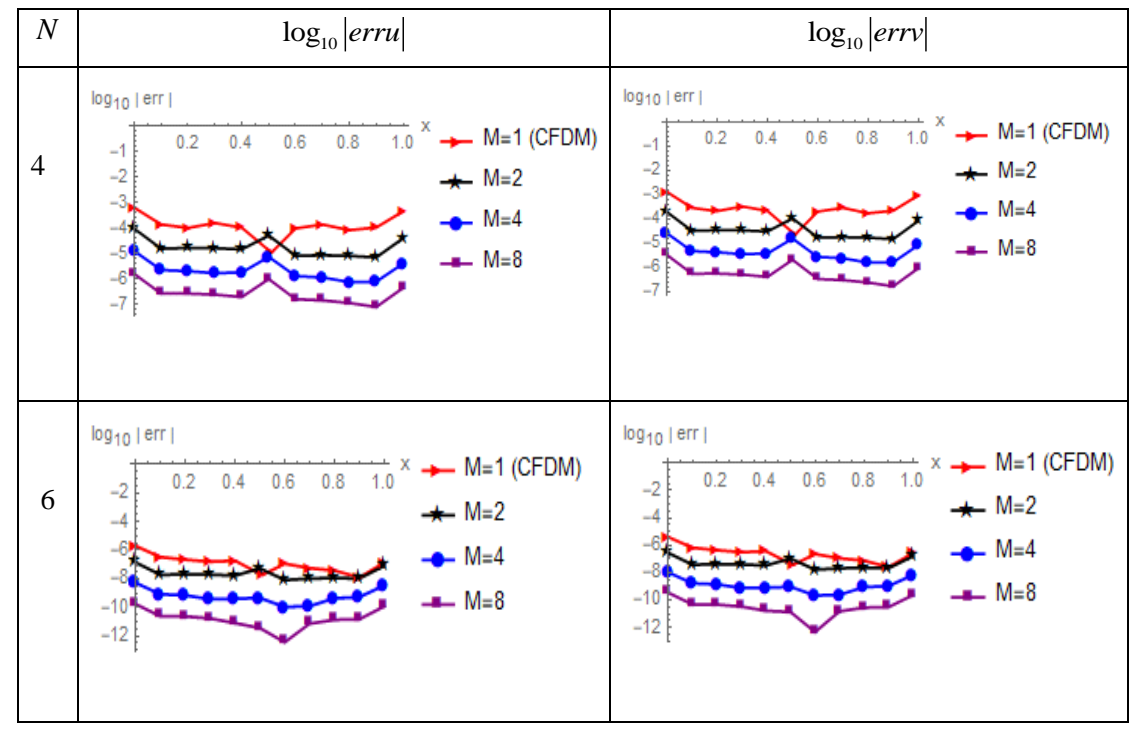

Similar to the first example, even for comparison of the present method with $[6,10,25]$, the better results are obtained.

\section{Conclusions}

In this paper, we proposed a new method, Smooth Composite CFDM which is based on CFDM and it is more effective than CFDM. The superiority of the presented method comes from taking into account the first order smoothness at the end points of the subintervals, which is distinctive property from CFDM and Composite CFDM. Convergence and error analyses for the method are investigated. The method is used for the solution of the coupled Lane Emden boundary value problems arising in the Catalytic Diffusion Reactions. The numerical results reveal that this new technique, i.e. Smooth Composite CFDM achieves higher accuracy, less calculation and high efficiency for this type of problems. 


\section{References}

[1] J. H. Lane, On theoretical temperature of the sun under the hypothesis of a gaseous mass maintaining its internal heat and depending on the laws of gases known to terrestrial experiment, Am. J. Sci. Arts. Ser. 2 (1870) 57-74.

[2] R. Emden, Gaskugeln Anwendungen der Mechan. Warmtheorie. Teubner, Leipzig, 1907.

[3] R. C. Duggan, A. M. Goodman, Pointwise bounds for a nonlinear heat conduction model of the human head, Bull. Math. Biol. 48 (1986) 229-236.

[4] K. Reger, R. A. Van Gorder, Lane-Emden equations of second kind modelling thermal explosion in infinite cylinder and sphere, Appl. Math. Mech. 34 (2013) 1439-1452.

[5] A. Wazwaz, Solving the non-isothermal reaction-diffusion model equations in a spherical catalyst by the variational iteration method, Chem. Phys. Lett. 679 (2017) 132136.

[6] R. Rach, J. S. Duan, A. M. Wazwaz, Solving coupled Lane-Emden boundary value problems in catalytic diffusion reactions by the Adomian decomposition method, $J$. Math. Chem. 52 (2014) 255-267.

[7] R. Singh, Optimal homotopy analysis method for the non-isothermal reaction-diffusion model equations in a spherical catalyst, J. Math. Chem. 56 (2018) 2579-2590.

[8] S. Aydinlik, A. Kiris, A high-order numerical method for solving nonlinear LaneEmden type equations arising in astrophysics, Astrophys. Space Sci. 363 (2018) \#264.

[9] T. C. Hao, F. Z. Cong, Y. F. Shang, An efficient method for solving coupled LaneEmden boundary value problems in catalytic diffusion reactions and error estimate, $J$. Math. Chem. 56 (2018) 2691-2706.

[10] R. Singh, A. M. Wazwaz, An efficient algorithm for solving coupled Lane-Emden boundary value problems in catalytic diffusion reactions: The homotopy analysis method, MATCH Commun. Math. Comput. Chem. 81 (2019) 785-800.

[11] R. Singh, H. Garg, V. Guleria, Haar wavelet collocation method for Lane-Emden equations with Dirichlet, Neumann and Neumann-Robin boundary conditions, J. Comp. Appl. Math. 346 (2019) 150-161.

[12] P. Roul, K. Thula, A fourth-order B-spline collocation method and its error analysis for Bratu-type and Lane-Emden problems, Int. J. Comput. Math. 96 (2019) 85-104.

[13] H. Madduri, P. Roul, A fast-converging iterative scheme for solving a system of LaneEmden equations arising in catalytic diffusion reactions, J. Math. Chem. 57 (2019) 570582.

[14] J. Shahni, R. Singh, An efficient numerical technique for Lane-Emden-Fowler boundary value problems: Bernstein collocation method, Eur. Phys. J. Plus. 135 (2020) \#475. 
[15] M. P. Alam, T. Begum, A. Khan, A new spline algorithm for solving non-isothermal reaction diffusion model, Chem. Phys. Lett. 754 (2020) \#137651.

[16] G. Sevin, Taylor wavelet solution of linear and nonlinear Lane-Emden equations, Appl. Num. Math. 158 (2020) 44-53.

[17] A. Saadatmandi, M. Dehghan, The numerical solution of problems in calculus of variation using Chebyshev finite difference method, Physics Lett. A. 372 (2008) 40374040 .

[18] H. Azizi, G. B. Loghmani, numerical approximation for space fractional diffusion equations via Chebyshev finite difference method, J. Fract. Calc. Appl. 4 (2013) 303311.

[19] H. Azizi, Chebyshev finite difference method for fractional boundary value problems, J. Math. Ext. 9 (2015) 57-71.

[20] A. Saadatmandi, S. Fayyaz, Chebyshev finite difference method for solving a mathematical model arising in wastewater treatment plants. Comp. Meth. Diff. Eq. 6 (2018) 448-455.

[21] H. R. Marzban, S. M. Hoseini, Solution of linear optimal control problems with time delay using a composite Chebyshev finite difference method. Optim. Control Appl. Methods 34 (2013) 253-274.

[22] E. M. E. Elbarbary, M. El-Kady, Chebyshev finite difference approximation for the boundary value problems, Appl. Math. Comp. 139 (2003) 513-523.

[23] E. M. E. Elbarbary, S. M. El-Sayed, Higher order pseudospectral differentiation matrices, App. Num. Math. 55 (2005) 425-438.

[24] C. Canuto, A. Quarteroni, M. Y. Hussaini, T. A. Zang, Spectral Methods Fundamentals in Single Domains, Springer-Verlag, Berlin, 2006.

[25] M. Turkyilmazoglu, Effective computation of exact and analytic approximate solutions to singular nonlinear equations of Lane-Emden-Fowler type, Appl. Math. Model. 37 (2013) 7539-7548. 